\title{
On the behavior in time of solutions to motion of Non-Newtonian fluids
}

\author{
Gioconda Moscariello and Maria Michaela Porzio@
}

\begin{abstract}
We study the behavior on time of weak solutions to the nonstationary motion of an incompressible fluid with shear rate dependent viscosity in bounded domains when the initial velocity $u_{0} \in L^{2}$. Our estimates show the different behavior of the solution as the growth condition of the stress tensor varies. In the "dilatant" or "shear thickening" case we prove that the decay rate does not depend on $u_{0}$, then our estimates also apply for irregular initial velocity.
\end{abstract}

Mathematics Subject Classification. 76D03, 35Q35, 76D07 .

Keywords. Decay estimates, Non-Newtonian fluids, Dirichlet boundary initial value problems, weak solutions.

\section{Introduction}

Let $\Omega$ be a bounded open set of $\mathbb{R}^{N}, N>2$. For $0<T<+\infty$ we set $\Omega_{T}=\Omega \times(0, T)$. The unsteady motion of an incompressible fluid with sheardependent viscosity in a cylinder $\Omega_{T}$ is governed by the conservation of volume and the conservation of momentum equations

$$
\begin{aligned}
\nabla \cdot u & =0 \quad \text { in } \quad \Omega_{T} \\
\partial_{t} u+\nabla \cdot(u \otimes u-\mathbf{S}+p \mathbf{I}) & =-\nabla \cdot f \quad \text { in } \quad \Omega_{T}
\end{aligned}
$$

where $\nabla \cdot u \equiv \partial_{x_{i}} u^{i}$ and we use the notation that repeated subscripts imply summation over 1 to $N$. Here

$$
\begin{array}{ll}
\mathbf{S}=\left\{S_{i j}\right\} & \text { is the deviatoric stress tensor } \\
p & \text { is the pressure, } \\
u=\left\{u^{1}, \ldots u^{N}\right\} & \text { is the velocity }, \\
f=\left\{f_{i, j}\right\} & \text { is the external force tensor } .
\end{array}
$$

We assume that at the initial time $t=0$, the initial velocity is a vector field $u_{0}$

$$
u(0)=u_{0} \quad \text { in } \quad \Omega
$$


with

$$
\nabla \cdot u_{0}=0
$$

and on the boundary the following aderence's condition holds

$$
u=0 \quad \text { on } \partial \Omega \times(0, T)
$$

The stress tensor $\mathbf{S}$ may depend on both $(x, t)$ and the "rate of strain tensor" $D=\left\{D_{i, j}\right\}$, which is defined by

$$
D_{i, j}=D_{i, j}(u) \equiv \frac{1}{2}\left(\partial_{x_{j}} u^{i}+\partial_{x_{i}} u^{j}\right), \quad i, j=1, \ldots N .
$$

For a background on Non-Newtonian fluids we refer to $[1,2,9]$ and the references therein.

The model (1.1)-(1.4) has been considered by J. Wolf in [21] and by L. Diening, M. R $\dot{u} \ddot{z}$ ička and J. Wolf in [5] to describe the motion of fluid u through $\Omega$.

The fluids with "shear dependent viscosity" are given by the constitutive law

where $^{1}$

$$
\mathbf{S}=\nu\left(D_{I I}\right) D
$$

$$
D_{I I}=\frac{1}{2} D: D=\text { second invariant of } D
$$

and $\nu$ is the generalized viscosity function.

As noticed in [5] the model (1.5) includes all power-law and Carreautype models, which are quite popular among rheologists. Such models are used in many areas of engineering sciences such as chemical engineering, colloidal mechanics, glaciology, geology and blood rheology (see [11] for a discussion of such models and further references).

Special cases of constitutive laws are the following

$$
\mathbf{S}=\nu_{0}\left(D_{I I}\right)^{\frac{q-2}{2}} D
$$

and

$$
\mathbf{S}=\nu_{0}\left[1+\left(D_{I I}\right)^{\frac{q-2}{2}}\right] D,
$$

where the constants $\nu_{0}$ and $q$ satisfy

$$
\nu_{0}>0, \quad 1<q<+\infty,
$$

(see $[1,3,20]$ and the references therein). A fluid which is determined by the first of these constitutive laws is said

$$
\begin{array}{ll}
\text { "dilatant" or "shear thickening" } & \text { if } q>2 \\
\text { "Newtonian" } & \text { if } q=2 \\
\text { "pseudoplastic" or "shear thinning" } & \text { if } 1<q<2 .
\end{array}
$$

Let us denote by $M_{s y m}^{N}$ the vector space of the symmetric $N \times N$ matrices $\xi=\left\{\xi_{i j}\right\}$ equipped with the scalar product $\xi: \eta$ and with the inducted norm

\footnotetext{
${ }^{1}$ Here, and throughout what follows, for two matrices $\mathbf{A}$ and $\mathbf{B}$ of $R^{N^{2}}$, we denote by $A: B$ the sum $A_{i j} B_{i j}$.
} 
$\|\xi\| \equiv(\xi: \xi)^{\frac{1}{2}}$. Moreover, let $a \cdot b$ be the Euclidean scalar product in $\mathbb{R}^{N}$ and by $|a|$ the inducted Euclidean norm.

We assume that the the deviatoric stress $\mathbf{S}: \Omega_{T} \times M_{\text {sym }}^{N} \rightarrow M_{\text {sym }}^{N}$ is a Caratheodory function satisfying for every $\xi$ and $\eta$ in $M_{s y m}^{N}$ and for almost every $(x, t) \in \Omega_{T}$

$$
\begin{aligned}
& \|\mathbf{S}(x, t, \xi)\| \leq c_{0}\|\xi\|^{q-1}+g_{1} \\
& \mathbf{S}(x, t, \xi): \xi \geq \nu_{0}\|\xi\|^{q}-g_{2}
\end{aligned}
$$

where $\nu_{0}$ and $c_{0}$ are positive constants, and $g_{i}(i=1,2)$ are non negative functions satisfying $g_{1} \in L^{q^{\prime}}\left(\Omega_{T}\right)$ and $g_{2} \in L^{1}\left(\Omega_{T}\right)$.

Before recalling what we mean here by weak solutions to (1.1)-(1.4), we recall some function spaces we need.

Here, $C_{0}^{\infty}(\Omega)$ denotes the space of all smooth functions having compact support in $\Omega, W^{k, q}(\Omega)(k \in \mathbf{N}$ and $1 \leq q \leq+\infty)$ are the usual Sobolev spaces and $W_{0}^{k, q}(\Omega)$ is the closure of $C_{0}^{\infty}(\Omega)$ in $W^{k, q}(\Omega)$.

If $\left(X,\|\cdot\|_{X}\right)$ is a normed space, by $L^{q}(0, T ; X)$ we denote the space of all the Bochner measurable functions $\varphi:(0, T) \rightarrow X$ such that

$$
\begin{aligned}
\|\varphi\|_{L^{q}(0, T ; X)} & =\left(\int_{0}^{T}\|\varphi(t)\|_{X}^{q} d t\right)^{\frac{1}{q}}<+\infty \quad \text { if } 1 \leq q<+\infty \\
\|\varphi\|_{L^{\infty}(0, T ; X)} & =\operatorname{ess}_{\sup _{t \in(0, T)}\|\varphi(t)\|_{X}<+\infty} \quad \text { if } q=+\infty
\end{aligned}
$$

Finally, we denote

$H_{q}$ the closure of the set $\left\{\varphi \in C_{0}^{\infty}(\Omega)^{N}: \nabla \cdot \varphi=0\right\}$ respect to the norm of $L^{q}(\Omega)^{N}$ $V_{q}$ is the closure of the set $\left\{\varphi \in C_{0}^{\infty}(\Omega)^{N}: \nabla \cdot \varphi=0\right\}$ respect to the norm

$$
\|\varphi\|_{V_{q}}=\|D(\varphi)\|_{L^{q}(\Omega)} .
$$

To simplify the notation, for $q=2$ we use just notation $\mathrm{H}$ for the space $\mathrm{H}_{2}$ and we will not distinguish between the norm of scalar-valued, vector-valued or tensor-valued versions of all the spaces defined above.

Finally, $|\Omega|$ denotes the Lebesgue measure of $\Omega$.

We recall that since we are assuming $\Omega$ bounded, by means of Korn's inequality there exists a positive constant $\gamma_{0}$, such that

$$
\|v\|_{W^{1, q}(\Omega)} \leq \gamma_{0}\|v\|_{V_{q}} \quad \text { for every } \quad v \in V_{q} .
$$

Definition 1.1. Assume (1.8)-(1.9) with $\frac{2 N}{N+2} \leq q<+\infty$. Let $f$ be in $\left(L^{q^{\prime}}\left(\Omega_{T}\right)\right)^{N^{2}}$ and $u_{0} \in H$. A weak solution of $(1.1)-(1.4)$ is a vector-valued function $u \in L^{q}\left(0, T ; V_{q}\right) \cap L^{\infty}(0, T ; H)$ satisfying

$$
\begin{gathered}
-\int_{\Omega_{T}} u \cdot \partial_{t} \varphi d x d t+\int_{\Omega_{T}}(\mathbf{S}(x, t, D(u))-u \otimes u): D(\varphi) d x d t \\
=\int_{\Omega_{T}} f: \nabla \varphi d x d t+\int_{\Omega_{T}} u_{0} \cdot \varphi(0) d x
\end{gathered}
$$

for every $\varphi \in C^{\infty}\left(\Omega_{T}\right)^{N}$ with $\nabla \cdot \varphi=0$ and $\operatorname{supp}(\varphi) \subset \subset \Omega \times[0, T)$. 
Thanks to the parabolic embedding, any weak solution $u$ belongs to $L^{q \frac{N+2}{N}}\left(\Omega_{T}\right)$.

The assumption $q \geq \frac{2 N}{N+2}$ guarantees that $\mathrm{u}$ is in $L^{2}\left(\Omega_{T}\right)$.

Assuming that the external force verifies

$$
f \in\left(L^{q^{\prime}}\left(\Omega_{T}\right)\right)^{N^{2}},
$$

and the monotonicity condition

$$
(\mathbf{S}(x, t, \xi)-\mathbf{S}(x, t, \eta)):(\xi-\eta)>0 \quad \xi \neq \eta,
$$

the first results on existence of solutions were proved by O. Ladyzhenskaya in [8] and by J.L. Lions in [10] under the restriction that

$$
q \geq \frac{3 N+2}{N+2} .
$$

The uniqueness holds if $q>\frac{N+2}{2}$.

For $N=3$ and $2 \leq q<\frac{11}{5}$ the existence of a weak solution was proved by J. Malek, J. Nečas and M. R $\dot{u} \breve{z i}$ čka in [12] under further regularity assumptions on the domain and on $\mathbf{S}$.

Then, J. Wolf has completed the previous existence results in [21], covering the set

$$
2 \frac{N+1}{N+2}<q<+\infty .
$$

Finally, L. Diening, M. Rüüička and J. Wolf in [5] considered all the set

$$
\frac{2 N}{N+2}<q<+\infty
$$

by proving the existence of a weak solution $u \in C_{w}([0, T] ; H)$. We recall that a function $v \in L^{\infty}(0, T ; H)$ belongs to the function space $C_{w}([0, T] ; H)$ of weakly continuous functions from $[0, T]$ to $H$ if $v(\cdot, t) \in H$ for any $t \in[0, T]$ and

$$
t \longmapsto<\psi, v(t)>_{H} \quad \text { is continuous for any } \psi \in H^{\prime} .
$$

Here $<\cdot, \cdot>_{H}$ denotes the duality pairing between $H^{\prime}$ and $H$.

Solutions found in [5] and [21] are limit of functions solving approximating problems more regular than (1.1)-(1.4). Moreover these functions satisfy a balance energy equality (see also [4]).

Motivated by this topic we state the following

Definition 1.2. Let $f$ be in $\left(L^{q^{\prime}}\left(\Omega_{T}\right)\right)^{N^{2}}$ and $u_{0} \in H$. A weak solution $u \in$ $L^{q}\left(0, T ; V_{q}\right) \cap L^{\infty}(0, T ; H)$ to $(1.1)-(1.4)$ is a "weak energy" solution if there exists a sequence $u_{\varepsilon} \in L^{q}\left(0, T ; V_{q}\right) \cap L^{\infty}(0, T ; H)$ such that

$$
\begin{aligned}
& u_{\varepsilon}(t) \rightarrow u(t) \quad \text { in } H \text { for a.e. } 0 \leq t \leq T, \\
& \quad \frac{1}{2}\left\|u_{\varepsilon}(t)\right\|_{H}^{2}+\int_{0}^{t} \int_{\Omega} \mathbf{S}\left(x, s, D\left(u_{\varepsilon}\right)\right): D\left(u_{\varepsilon}\right) d x d s \\
& =\frac{1}{2}\left\|u_{0}\right\|_{H}^{2}+\int_{0}^{t} \int_{\Omega} f: \nabla u_{\varepsilon} d x d s \quad \text { for every } 0 \leq t \leq T .
\end{aligned}
$$


Let us remark that if a weak solution is bounded in $\Omega_{T}$, then it is a weak energy solution. Indeed (up to a regularization procedure) by using $u$ as test function in (1.11), integration by parts gives that

$$
\int_{0}^{t} \int_{\Omega}(u \otimes u): D(u) d x d s=0
$$

since $\nabla \cdot u=0$ and hence (1.15) follows with $u_{\varepsilon} \equiv u$.

If (1.13) holds true, thanks to the results of [5] and [21], for

$$
\frac{2 N}{N+2}<q<+\infty
$$

problem (1.1)-(1.4) admits a weak energy solution $u \in C_{w}([0, T] ; H)$.

Aim of this paper is to describe the behavior in time of weak energy solutions to (1.1)-(1.4).

Based on the Fourier splitting method, estimates on time of the solutions of Navier-Stokes equation and of non-Newtonian fluid were derived by M. E. Schonbek in [19] and by S. Nečasova and P. Penel in [14], respectively, when the spatial variable belongs to $\mathbb{R}^{N}$. Time decay estimates were also discussed in [4] when the spatial variable is in $\mathbb{R}_{+}^{N}$ and the Fourier splitting method does not apply. The solutions considered there are energy solutions in the sense of Definition 1.2.

The model treated by the previous authors concerns the case in which the deviatoric stress tensor $\mathbf{S}$ is given by (1.7) with particular values of $\mathrm{q}$.

Here we consider time decay estimates for a weak energy solution to general problem (1.1)-(1.4) having the spatial variable in a bounded domain $\Omega$ and in all cases in which this problem is known to be well posed.

Our estimates are based on an approach that highlights the different behavior as the exponent q varies.

To understand the large behavior of a viscous incompressible fluid we assume that conditions (1.8) and (1.9) hold for a.e. $(x, t) \in \Omega_{\infty} \equiv \Omega \times(0,+\infty)$ with $g_{1} \in L^{q^{\prime}}\left(\Omega_{T}\right), g_{2} \in L^{1}\left(\Omega_{T}\right)$ for every $T>0$ and

$$
f \in\left(L^{q^{\prime}}\left(\Omega_{\infty}\right)\right)^{N^{2}} .
$$

In this case we say that $u \in L_{\text {loc }}^{q}\left(0,+\infty ; V_{q}\right) \cap L_{l o c}^{\infty}(0,+\infty ; H)$ is a global weak energy solution to (1.1)-(1.4) if for any $T>0 \mathrm{u}$ is a weak energy solution in $\Omega_{T}$ according to Definition 1.2 above.

Our main result is the following.

Theorem 1.1. Assume (1.8), (1.9) and (1.16). Let $u$ be a global weak energy solution to (1.1)-(1.4). If $g_{2} \in L^{1}\left(\Omega_{\infty}\right)$ then $u \in C_{w}([0, T] ; H)$ for any $T>0$ and the following estimates hold true for any $t>0$

$$
\begin{gathered}
\|u(t)\|_{L^{2}(\Omega)}^{2} \leq \Lambda_{0} e^{-B t}+\int_{\frac{t}{2}}^{t} g(s) d s \quad \text { if } q \leq 2 \\
\|u(t)\|_{L^{2}(\Omega)}^{2} \leq \frac{\Lambda_{1}}{t^{\frac{1}{2}-1}}+\int_{\frac{t}{2}}^{t} g(s) d s \quad \text { if } q>2
\end{gathered}
$$


where

$$
g(s) \equiv 2\left[c\left(q, \nu_{0}, \gamma_{0}\right)\|f(s)\|_{L^{q^{\prime}}(\Omega)}^{q^{\prime}}+\left\|g_{2}(s)\right\|_{L^{1}(\Omega)}\right]
$$

[with $\gamma_{0}$ as in (1.10)]

$$
\begin{aligned}
& \Lambda_{0}=\left\|u_{0}\right\|_{H}^{2}+\|g(s)\|_{L^{1}((0,+\infty))}, \\
& \Lambda_{1}=\left[\frac{\left(\frac{q}{2}-1\right) c_{1}}{2|\Omega|^{\gamma}}\right]^{-\frac{1}{2}-1}
\end{aligned}
$$

$c_{1}=c_{1}\left(\nu_{0}, \gamma_{0}, q, N\right)$ (see formula $(2.7)$ ) and $B=B\left(\Omega, \Lambda_{0}, q, N, \nu_{0}\right)$.

An estimate of the constant $B$ is given in formula (3.16) below.

The existence of a global weak energy solution to (1.1)-(1.4) is proved in Sect. 2.2.

We point out that the previous result shows that although the global weak energy solution $u$ decays in time for every value of $q$, there is a deep difference in the decay estimates between the "dilatant" or "shear thickening" case $q>2$ and all the other cases ( "Newtonian" or "shear Thinning"). As a matter of fact, if $q>2$, all the estimates in Theorem 1.1 are universal estimates, i.e., do not depend on the initial value $u_{0}$. In other words, in the "dilatant" or "shear thickening" case the initial velocity does not influence at all the behavior in time of the velocity of the fluid.

Thanks to this argument, when $q>2$, we are able to provide the existence of a suitable weak solution also when $u_{0}$ is just a summable function (see Sect. 4).

Moreover, we can show that, although the initial datum is not regular, as soon as $t>0$ the solution we found has the same regularity and decay behavior of the case in which $u_{0}$ is regular.

In the particular case that the deviatoric stress tensor $\mathbf{S}$ is given by (1.7) we show that an exponential decay estimate holds true regardless of the growth exponent q (see Proposition 3.1 below).

We point out that in unbounded domains optimal algebraic estimates have been proved in [14] and [4]. Here we reach an exponential decay rate thanks to the boundedness of the domain $\Omega$.

The paper is organized as follows: in Sect. 2 we recall some known results that will be an essential tool in proving our decay estimates (see Sect. 2.1) and we construct a weak global energy solution (see Sect. 2.2). In Sect. 3.1 we prove Theorem 1.1 and we study the particular case (1.7). Finally, in Sect. 4 we study the case of not regular initial data.

\section{Preliminary results}

As recalled in the Introduction, for the convenience of the reader we recall here some known results that will be an essential tool in proving the decay estimates stated in the previous section. 


\subsection{Gronwall type Lemmas}

Lemma 2.1. Assume $T \in(0,+\infty]$ and let $\phi(t)$ a continuous and non negative function defined in $[0, T)$ and verifying

$$
\phi\left(t_{2}\right)-\phi\left(t_{1}\right)+M \int_{t_{1}}^{t_{2}} \phi(t)^{1+\nu} d t \leq \int_{t_{1}}^{t_{2}} g(t) d t
$$

for every $0 \leq t_{1} \leq t_{2}<T$ where $M$ and $\nu$ are positive constants and $g$ is a non negative function in $L_{\text {loc }}^{1}([0, T))$. Then it results

$$
\phi(t) \leq \frac{\phi(0)}{\left[1+\nu M \phi(0)^{\nu} t\right]^{\frac{1}{\nu}}}+\int_{0}^{t} g(s) d s \quad \text { for every } t \in[0, T) .
$$

Moreover, the following estimate holds

$$
\phi(t) \leq \frac{M_{0}}{t^{\frac{1}{\nu}}}+\int_{\frac{t}{2}}^{t} g(s) d s \quad \text { for every } t \in(0, T)
$$

where $M_{0}=\left(\frac{\nu M}{2}\right)^{-\frac{1}{\nu}}$.

The previous Lemma can be easily proved by a straightforward modification of the proof of Proposition 3.1 of [13] and hence we omit it.

Lemma 2.2. Assume $T \in(0,+\infty]$ and let $\phi(t)$ a continuous and non negative function defined in $[0, T)$ verifying

$$
\phi\left(t_{2}\right)-\phi\left(t_{1}\right)+M \int_{t_{1}}^{t_{2}} \phi(t) d t \leq \int_{t_{1}}^{t_{2}} g(t) d t
$$

for every $0 \leq t_{1} \leq t_{2}<T$ where $M$ is a positive constant and $g$ is a non negative function in $L_{l o c}^{1}([0, T))$. Then it results

$$
\phi(t) \leq \phi(0) e^{-M t}+\int_{0}^{t} g(s) d s \quad \text { for every }>0 .
$$

Moreover, if $T=+\infty$ and $g$ belongs to $L^{1}((0,+\infty))$ we have

$$
\phi(t) \leq \Lambda e^{-\frac{M}{2} t}+\int_{\frac{t}{2}}^{t} g(s) d s \quad \text { for every } t>0,
$$

where

$$
\Lambda=\phi(0)+\int_{0}^{+\infty} g(s) d s .
$$

In particular, we get that

$$
\lim _{t \rightarrow+\infty} \phi(t)=0 .
$$

We omit the proof of the previous Lemma since it is an obvious modification of the proof of Proposition 3.2 of [13].

Remark 2.1. Previous Lemmas have been used in [13] and [7] to study asymptotic estimates for evolution problems. Analogous techniques, developed in [15] and in [17], have been useful also for other parabolic problems (see [16] and $[18])$. 


\subsection{Existence of a global weak energy solution}

In this subsection we prove the existence of a global weak energy solution to problem (1.1)-(1.4). The existence of a weak energy solution can be deduced from [21] and [5]. Here, for convenience of the reader, we state the main tools of the construction in $\Omega_{T}$, in order to obtain a global weak energy solution.

Proposition 2.1. Assume that the structural assumptions (1.8), (1.9) and (1.13) hold true a.e. in $\Omega_{\infty}$ with $q>\frac{2 N}{N+2}, g_{2} \in L^{1}\left(\Omega_{\infty}\right)$ and $g_{1} \in L^{q^{\prime}}\left(\Omega_{T}\right)$ for any $T>0$. Let $f \in\left(L^{q^{\prime}}\left(\Omega_{\infty}\right)\right)^{N^{2}}$ and $u_{0} \in H$. Then there exists a weak global energy solution $u$ of $(1.1)-(1.4)$.

Moreover, $u \in C_{w}([0, T] ; H)$ for any $T>0$.

Proof. As in [5] and [21] we consider for any $\varepsilon>0$ and for any $T>0$ the following problem

$$
\begin{cases}\nabla \cdot u_{\varepsilon}=0 & \text { in } \Omega_{T}, \\ \partial_{t} u_{\varepsilon}+\nabla \cdot\left(u_{\varepsilon} \otimes u_{\varepsilon} \psi_{\varepsilon}\left(\left|u_{\varepsilon}\right|\right)-\mathbf{S}\left(x, t, D\left(u_{\varepsilon}\right)\right)+f\right)=-\nabla p_{\varepsilon} & \text { in } \Omega_{T}, \\ u_{\varepsilon}=0 & \text { on } \partial \Omega \times(0, T), \\ u_{\varepsilon}(0)=u_{0} & \text { in } \Omega,\end{cases}
$$

where

$$
\psi_{\varepsilon}(\tau) \equiv \psi(\varepsilon \tau), \quad \tau \in[0,+\infty)
$$

with $\psi \in C^{\infty}([0,+\infty))$ a non-increasing function such that

$$
\begin{aligned}
& 0 \leq \psi \leq 1 \text { in }[0,+\infty) \phi \equiv 1 \text { in }[0,1] \\
& \psi \equiv 0 \text { in }[2,+\infty] \quad 0 \leq-\psi^{\prime} \leq 2 .
\end{aligned}
$$

Here, by a solution of $(2.6)$ we mean a function $u_{\varepsilon} \in L^{\infty}(0, T ; H) \cap L^{q}\left(0, T ; V_{q}\right)$ satisfying

$$
\begin{aligned}
& -\int_{\Omega_{T}} u_{\varepsilon} \cdot \partial_{t} \varphi d x d t+\int_{\Omega_{T}}\left(\mathbf{S}\left(x, t, D\left(u_{\varepsilon}\right)\right)-u_{\varepsilon} \otimes u_{\varepsilon} \psi_{\varepsilon}\left(\left|u_{\varepsilon}\right|\right)\right): D(\varphi) d x d t \\
& \quad=\int_{\Omega_{T}} f: \nabla \varphi d x d t+\int_{\Omega_{T}} u_{0} \cdot \varphi(0) d x
\end{aligned}
$$

for every $\varphi \in C^{\infty}\left(\Omega_{T}\right)^{N}$ with $\nabla \cdot \varphi=0$ and $\operatorname{supp}(\varphi) \subset \subset \Omega \times[0, T)$.

By using fixed point Theorem in [21] it is proved that, for any $\varepsilon>$ 0 , problem (2.6) admits a unique solution $u_{\varepsilon} \in C([0, T] ; H) \cap L^{q}\left(0, T ; V_{q}\right)$. Moreover this solution satisfies the following energy equality

$$
\begin{aligned}
& \frac{1}{2}\left\|u_{\varepsilon}(t)\right\|_{H}^{2}+\int_{0}^{t} \int_{\Omega} \mathbf{S}\left(x, s, D\left(u_{\varepsilon}\right)\right): D\left(u_{\varepsilon}\right) d x d s= \\
& \frac{1}{2}\left\|u_{0}\right\|_{H}^{2}+\int_{0}^{t} \int_{\Omega} f: \nabla u_{\varepsilon} d x d s \quad \text { for every } 0 \leq t \leq T
\end{aligned}
$$

(see Theorem 3.1 of [21]).

From (2.7), thanks to the coercivity condition (1.9) we get

$$
\left\|u_{\varepsilon}\right\|_{L^{\infty}(0, T ; H)}^{2}+\left\|D\left(u_{\varepsilon}\right)\right\|_{L^{q}\left(\Omega_{T}\right)}^{q} \leq c
$$


where $c$ here and in what follows denotes a positive constant, which may vary from line to line but does not depend on the parameter $\varepsilon$.

By virtue of Korn's inequality and Sobolev's embedding Theorem we get

$$
\left\|u_{\varepsilon}\right\|_{L^{q}\left(0, T ; W^{1, q}(\Omega)\right)}+\left\|u_{\varepsilon}\right\|_{L^{q}\left(0, T ; L^{q^{*}}(\Omega)\right)} \leq c
$$

where $q^{*}=\frac{q N}{N-q}$.

Then by Sobolev's inequalities and Holder's inequality from (2.8) to (2.9) we deduce that

$$
\left\|u_{\varepsilon}\right\|_{L^{q \frac{N+2}{N}}\left(\Omega_{T}\right)} \leq c .
$$

We recall that from the bound of $\mathrm{q}$ we have that

$$
q \frac{N+2}{N}>2 \text {. }
$$

Now we can pass to a subsequence, still denoted by $u_{\varepsilon}$ for simplicity, and find $u \in L^{q}\left(0, T ; V_{q}\right) \cap L^{\infty}(0, T ; H)$ such that

$$
u_{\varepsilon} \rightarrow u \quad \text { weakly in } L^{q \frac{N+2}{N}}\left(\Omega_{T}\right)
$$

Arguing as in Section 4 of [5], it is possible to prove that

$$
u \in C_{w}([0, T] ; H) \text { and } u(0)=u_{0},
$$

and

$$
u_{\varepsilon} \rightarrow u \text { strongly in } L^{r}\left(0, T ; L^{2}(G)\right), 1 \leq r<\infty,
$$

where $G \subset \subset \Omega$ is a arbitrary open bounded set.

Then for a.e. $t \in[0, T]$

$$
u_{\varepsilon}(\cdot, t) \rightarrow u(\cdot, t) \quad \text { a.e. in } \Omega
$$

and so from (2.8) for a.e. $t \in[0, T]$

$$
u_{\varepsilon}(\cdot, t) \rightarrow u(\cdot, t) \quad \text { weakly in } \mathrm{H} .
$$

Finally since $u \in C_{w}([0, T], H)$ we get that for any $t \in[0, T]$

$$
u_{\varepsilon}(\cdot, t) \rightarrow u(\cdot, t) \quad \text { weakly in } \mathrm{H} \text {. }
$$

It is now easy to prove (see [5] and [21]) that the function $u$ constructed as above satisfies (1.11) and so it is a weak energy solution to (1.1)-(1.4) (i.e. a weak energy solution in $\Omega_{T}$ ). Now we prove the existence of a global weak energy solution.

We notice that, since by construction $u_{\varepsilon}$ is the unique solution of (2.6) which belongs to $C([0, T] ; H) \cap L^{q}\left(0, T ; V_{q}\right)$, it is possible to extend it (in a unique way) to a global solution of $(2.6)$ belonging to $C_{l o c}([0,+\infty) ; H) \cap$ $L_{l o c}^{q}\left([0,+\infty) ; V_{q}\right)$. We denote such a global solution again $u_{\varepsilon}$.

Hence, let $T_{0}>0$ arbitrarily fixed. As recalled above, there exists a subsequence of $u_{\varepsilon}$, that we denote $u_{\varepsilon_{0}}$ that converges to a weak energy solution $u$ of $(1.1)-(1.4)$ in $\Omega \times\left(0, T_{0}\right)$ (i.e. $u \in C_{w}\left(\left[0, T_{0}\right] ; H\right)$ is a weak energy solution of (1.1)-(1.4) with $T$ replaced by $\left.T_{0}\right)$. Being $u_{\varepsilon_{0}}$ a subsequence of $u_{\varepsilon}$, it follows that it is a sequence of global solutions. In particular, $u_{\varepsilon_{0}}$ is a sequence of solutions of $(2.6)$ in $\Omega \times\left(0,2 T_{0}\right)$ which belongs to $C\left(\left[0,2 T_{0}\right] ; H\right) \cap L^{q}\left(0,2 T_{0} ; V_{q}\right)$. 
Thus, there exists a subsequence of $u_{\varepsilon_{0}}$, that we denote with $u_{\varepsilon_{1}}$, that converges to a weak energy solution $u_{1}$ of $(1.1)-(1.4)$ in $\Omega \times\left(0,2 T_{0}\right)$, i.e. $u_{1} \in$ $C_{w}\left(\left[0,2 T_{0}\right] ; H\right)$ is a weak energy solution of $(1.1)-(1.4)$ with $T$ replaced by $2 T_{0}$. By construction $u=u_{1}$ in $\Omega \times\left(0, T_{0}\right)$ and consequently we can denote $u=u_{1}$ in all the set $\Omega \times\left(0,2 T_{0}\right)$ and $u \in C_{w}\left(\left[0,2 T_{0}\right] ; H\right)$. By a recursive argument, we can construct a global energy solution $\mathrm{u}$ of (1.1)-(1.4) which belongs to $C_{w}([0, T] ; H)$ for every $T>0$.

\section{Decay estimates}

Aim of this section is to prove Theorem 1.1 and to treat the special case (1.7).

\subsection{Proof of Theorem 1.1}

Let $\mathrm{u}$ be a global weak energy solution to (1.1)-(1.4). We point out that $\mathrm{u}$ belongs to $C_{w}([0,+\infty) ; H)$ since any weak solution of $(1.1)-(1.4)$ belongs to $C_{w}([0, T] ; H)$. As a matter of fact, by $(1.11)$ it follows that $u_{t} \in L^{1}\left(0, T ; Y^{*}\right)$ where

$$
Y \equiv V_{q} \cap V_{\kappa} \cap H, \quad \text { where } \quad \kappa=\frac{q(N+2)}{q(N+2)-2 N} .
$$

Hence, u belongs to $C\left([0, T] ; Y^{*}\right)$ (see $[6]$ ). Now, to conclude that $\mathrm{u}$ is in $C_{w}([0, T] ; H)$ we follow the reasoning in [21]. Let $t_{0} \in[0, T]$ arbitrarily fixed. Since, $u$ belongs to $L^{\infty}(0, T ; H)$, there exists a sequence $t_{n} \in[0, T]$ such that $t_{n} \rightarrow t_{0}$ with $u\left(t_{n}\right)$ bounded in $\mathrm{H}$. Hence, by the reflexivity, there exists a subsequence $u\left(t_{n_{k}}\right)$ and $\xi \in H$ such that

$$
u\left(t_{n_{k}}\right) \rightarrow \xi \quad \text { weakly in } \mathrm{H} \quad \text { as } k \rightarrow+\infty .
$$

On the other hand, being $\mathrm{u}$ in $C\left([0, T] ; Y^{*}\right)$, it results

$$
u\left(t_{n_{k}}\right) \rightarrow u\left(t_{0}\right) \quad \text { strongly in } Y^{*} \quad \text { as } k \rightarrow+\infty .
$$

Recalling that $\mathrm{H}$ is continuously and densely imbedded into $Y^{*}$ it follows $\xi=u\left(t_{0}\right)$. This implies that $u\left(t_{n_{k}}\right)$ converges weakly to $u\left(t_{0}\right)$ in $\mathrm{H}$ as $k \rightarrow+\infty$. Whence u belongs to $C_{w}([0, T] ; H)$.

By definition, for every $T>0$ there exist $u_{\varepsilon} \in L^{q}\left(0, T ; V_{q}\right) \cap L^{\infty}(0, T ; H)$ satisfying (1.14) and (1.15). To prove estimate (1.17) it is sufficient to show that this estimate is satisfied for every $t \in(0, T)$ by the approximating solutions $u_{\varepsilon} \in C([0, T] ; H) \cap L^{q}\left(0, T ; V_{q}\right)$ since this implies (thanks to the arbitrary choice of $T$ ) that $u$ satisfies (1.17) for almost every $t>0$ and hence (thanks to the regularity $\left.C_{w}([0,+\infty) ; H)\right)$ for any $t>0$.

Hence, choosing $t=t_{2}$ in (1.15) and then choosing $t=t_{1}$ again in (1.15) and subtracting the equations obtained in this way we deduce that for every $0 \leq t_{1}<t_{2} \leq T$

$$
\begin{aligned}
& \frac{1}{2}\left\|u_{\varepsilon}\left(t_{2}\right)\right\|_{H}^{2}-\frac{1}{2}\left\|u_{\varepsilon}\left(t_{1}\right)\right\|_{H}^{2}+\int_{t_{1}}^{t_{2}} \int_{\Omega} S\left(x, s, D\left(u_{\varepsilon}\right)\right): D\left(u_{\varepsilon}\right) d x d s \\
& =\int_{t_{1}}^{t_{2}} \int_{\Omega} f: \nabla u_{\varepsilon} d x d s
\end{aligned}
$$


We estimate the integrals in the previous equality. Thanks to assumption (1.9) we obtain

$$
\int_{t_{1}}^{t_{2}} \int_{\Omega} S\left(x, s, D\left(u_{\varepsilon}\right)\right): D\left(u_{\varepsilon}\right) d x d s \geq \nu_{0} \int_{t_{1}}^{t_{2}}\left\|D\left(u_{\varepsilon}\right)\right\|_{L^{q}(\Omega)}^{q} d s-\left\|g_{2}\right\|_{L^{1}\left(\Omega \times\left(t_{1}, t_{2}\right)\right)} \text {. }
$$

It remains to estimate the last integral in (3.1). By (1.10) it results

$$
\int_{t_{1}}^{t_{2}} \int_{\Omega} f: \nabla u_{\varepsilon} d x d s \leq \frac{\nu_{0}}{2} \int_{t_{1}}^{t_{2}}\left\|D\left(u_{\varepsilon}\right)\right\|_{L^{q}(\Omega)}^{q} d s+c\left(q, \nu_{0}, \gamma_{0}\right) \int_{t_{1}}^{t_{2}}\|f\|_{L^{q^{\prime}}(\Omega)}^{q^{\prime}}
$$

Hence, using the previous estimates in (3.1) we obtain

$$
\begin{gathered}
\left\|u_{\varepsilon}\left(t_{2}\right)\right\|_{H}^{2}-\left\|u_{\varepsilon}\left(t_{1}\right)\right\|_{H}^{2}+\nu_{0} \int_{t_{1}}^{t_{2}}\left\|D\left(u_{\varepsilon}\right)\right\|_{L^{q}(\Omega)}^{q} d s \\
\quad \leq 2 \int_{t_{1}}^{t_{2}}\left[c\left(q, \nu_{0}, \gamma_{0}\right)\|f\|_{L^{q^{\prime}(\Omega)}}^{q^{\prime}}+\left\|g_{2}\right\|_{L^{1}(\Omega)}\right] d s
\end{gathered}
$$

Notice that being $\Omega$ bounded by (1.10) we get

$$
\int_{t_{1}}^{t_{2}}\left\|D\left(u_{\varepsilon}\right)\right\|_{L^{q}(\Omega)}^{q} d s \geq \gamma_{0}^{-q} \int_{t_{1}}^{t_{2}}\left\|u_{\varepsilon}\right\|_{W^{1, q}(\Omega)}^{q} d s .
$$

Hence, we deduce (using Sobolev inequality ${ }^{2}$ )

$$
\begin{aligned}
& \nu_{0} \int_{t_{1}}^{t_{2}}\left\|D\left(u_{\varepsilon}\right)\right\|_{L^{q}(\Omega)}^{q} d s \geq \nu_{0} \gamma_{0}^{-q} \int_{t_{1}}^{t_{2}}\left\|u_{\varepsilon}\right\|_{W^{1, q}(\Omega)}^{q} d s \\
& \quad \geq c_{1} \int_{t_{1}}^{t_{2}}\left\|u_{\varepsilon}\right\|_{L^{q^{*}}(\Omega)}^{q} d s
\end{aligned}
$$

where $c_{1}=\nu_{0} \gamma_{0}^{-q} c_{S o b}^{q}$. By (3.3) and (3.6) it follows

$$
\begin{aligned}
& \left\|u_{\varepsilon}\left(t_{2}\right)\right\|_{H}^{2}-\left\|u_{\varepsilon}\left(t_{1}\right)\right\|_{H}^{2}+c_{1} \int_{t_{1}}^{t_{2}}\left\|u_{\varepsilon}\right\|_{L^{q^{*}}(\Omega)}^{q} d s \\
& \leq 2 \int_{t_{1}}^{t_{2}}\left[c\left(q, \nu_{0}, \gamma_{0}\right)\|f\|_{L^{q^{\prime}(\Omega)}}^{q^{\prime}}+\left\|g_{2}\right\|_{L^{1}(\Omega)}\right] d s .
\end{aligned}
$$

We recall that

$$
\left\|u_{\varepsilon}(t)\right\|_{H}=\left\|u_{\varepsilon}(t)\right\|_{L^{2}(\Omega)} .
$$

Hence, since

$$
2<q^{*} \quad \Leftrightarrow \quad q>\frac{2 N}{N+2}
$$

it results

$$
\left\|u_{\varepsilon}\right\|_{L^{2}(\Omega)}^{q} \leq\left\|u_{\varepsilon}\right\|_{L^{q^{*}}(\Omega)}^{q}|\Omega|^{\gamma}
$$

${ }^{2}$ Sobolev inequality:

$$
c_{S o b}\|v\|_{L^{q^{*}}(\Omega)} \leq\|v\|_{W_{0}^{1, q}(\Omega)}, \quad \text { for every } v \in W_{0}^{1, q}(\Omega),
$$

where $c_{S o b}=c(q, N)$. 
where $\gamma=\frac{q}{2}+\frac{q}{N}-1$. Using the previous inequality in (3.7) we deduce (for every $\left.0 \leq t_{1}<t_{2} \leq T\right)$

$$
\left\|u_{\varepsilon}\left(t_{2}\right)\right\|_{L^{2}(\Omega)}^{2}-\left\|u_{\varepsilon}\left(t_{1}\right)\right\|_{L^{2}(\Omega)}^{2}+c_{1}|\Omega|^{-\gamma} \int_{t_{1}}^{t_{2}}\left\|u_{\varepsilon}\right\|_{L^{2}(\Omega)}^{q} d s \leq \int_{t_{1}}^{t_{2}} g(s) d s
$$

where we have set

$$
g(s) \equiv 2\left[c\left(q, \nu_{0}, \gamma_{0}\right)\|f\|_{L^{q^{\prime}}(\Omega)}^{q^{\prime}}+\left\|g_{2}\right\|_{L^{1}(\Omega)}\right]
$$

Now, to conclude the proof we distinguish the following three cases: $q=2$, $q>2$ and $q<2$.

If $q=2$, since we are assuming that $f \in\left(L^{q^{\prime}}\left(\Omega_{\infty}\right)\right)^{N^{2}}$ and $g_{2} \in L^{1}\left(\Omega_{\infty}\right)$ it follows that $g$ belongs to $L^{1}((0,+\infty))$. Hence, applying Lemma 2.2 we deduce that the following estimate holds true for every $t>0$

$$
\left\|u_{\varepsilon}(t)\right\|_{L^{2}(\Omega)}^{2} \leq \Lambda_{0} e^{-B_{0} t}+\int_{\frac{t}{2}}^{t} g(s) d s
$$

where

$$
\Lambda_{0}=\left\|u_{0}\right\|_{H}^{2}+\|g(s)\|_{L^{1}((0,+\infty))} \quad \text { and } \quad B_{0}=\frac{c_{1}}{2}|\Omega|^{-\gamma} .
$$

By (3.11) it follows estimate (1.17) when $q=2$.

If $q>2$ the inequality (3.9) allows to apply Lemma 2.1 and to conclude that the following estimate holds true for every $t>0$

$$
\left\|u_{\varepsilon}(t)\right\|_{L^{2}(\Omega)}^{2} \leq \frac{\Lambda_{1}}{t^{\frac{1}{2}-1}}+\int_{\frac{t}{2}}^{t} g(s) d s
$$

where $\mathrm{g}$ is as in (3.10) and

$$
\Lambda_{1}=\left[\frac{\left(\frac{q}{2}-1\right) c_{1}}{2|\Omega|^{\gamma}}\right]^{-\frac{1}{\frac{q}{2}-1}}
$$

By (3.13) it follows estimate (1.17) when $q>2$.

Finally, let us consider the case $q<2$. By (3.9) we get for every $t \in[0, T]$

$$
\left\|u_{\varepsilon}(t)\right\|_{L^{2}(\Omega)}^{2} \leq\left\|u_{0}\right\|_{L^{2}(\Omega)}^{2}+\int_{0}^{T} g(s) d s \leq \Lambda_{0}
$$

from which we deduce, for every $0 \leq t_{1}<t_{2} \leq T$,

$$
\left\|u_{\varepsilon}(t)\right\|_{L^{2}(\Omega)}^{q}=\frac{\left\|u_{\varepsilon}(t)\right\|_{L^{2}(\Omega)}^{2}}{\left\|u_{\varepsilon}(t)\right\|_{L^{2}(\Omega)}^{2-q}} \geq \frac{\left\|u_{\varepsilon}(t)\right\|_{L^{2}(\Omega)}^{2}}{\Lambda_{0}^{1-\frac{q}{2}}},
$$

where $\Lambda_{0}$ is the constant defined in (3.12). By the previous inequality and (3.9) we have

$$
\left\|u_{\varepsilon}\left(t_{2}\right)\right\|_{L^{2}(\Omega)}^{2}-\left\|u_{\varepsilon}\left(t_{1}\right)\right\|_{L^{2}(\Omega)}^{2}+c_{1}|\Omega|^{-\gamma} \Lambda_{0}^{\frac{q}{2}-1} \int_{t_{1}}^{t_{2}}\left\|u_{\varepsilon}(t)\right\|_{L^{2}(\Omega)}^{2} d s \leq \int_{t_{1}}^{t_{2}} g(s) d s .
$$


Hence, applying again Lemma 2.2 we obtain that for every $t>0$

$$
\left\|u_{\varepsilon}(t)\right\|_{L^{2}(\Omega)}^{2} \leq \Lambda_{0} e^{-B t}+\int_{\frac{t}{2}}^{t} g(s) d s
$$

where $B=\frac{c_{1}}{2}|\Omega|^{-\gamma} \Lambda_{0}^{\frac{q}{2}-1}$ from which the inequality (1.17) follows also in this case.

Remark 3.1. The proof of Theorem 1.1 shows that in estimate (1.17) the following choice of $\mathrm{B}$ is admissible

$$
B=\frac{c_{1}}{2}|\Omega|^{-\gamma} \Lambda_{0}^{\frac{q}{2}-1} \quad c_{1}=\nu_{0} c_{S o b}^{q} \gamma_{0}^{-q} \quad \gamma=\frac{q}{2}+\frac{q}{N}-1
$$

with $c_{S o b}$ the Sobolev's constant defined in (3.5) and $\gamma_{0}$ as in (1.10).

Remark 3.2. We notice that by (3.3) and (1.17) it follows that for every $0<$ $t_{1}<t_{2}<+\infty$ the following estimates hold true

$$
\begin{aligned}
& \int_{t_{1}}^{t_{2}}\|D u(t)\|_{L^{q}(\Omega)}^{q} \leq \nu_{0}^{-1}\left[\int_{\frac{t_{1}}{2}}^{t_{2}} g(s) d s+\Lambda_{0} e^{-B t_{1}}\right] \quad \text { if } q \leq 2 \\
& \int_{t_{1}}^{t_{2}}\|D u(t)\|_{L^{q}(\Omega)}^{q} \leq \nu_{0}^{-1}\left[\int_{\frac{t_{1}}{2}}^{t_{2}} g(s) d s+\frac{\Lambda_{1}}{t_{1}^{\frac{q}{2}-1}}\right] \quad \text { if } q>2 .
\end{aligned}
$$

We conclude this section studying the particular case (1.7).

\subsection{The special case (1.7)}

We prove the following result.

Proposition 3.1. Let $\mathbf{S}$ be as in (1.7), $q>\frac{2 N}{N+2}, f \in\left(L^{q^{\prime}}\left(\Omega_{\infty}\right)\right)^{N^{2}}$ and $u_{0} \in H$. Then every global weak energy solution $u$ of $(1.1)-(1.4)$ belongs to $C_{w}([0, T] ; H)$ for any $T>0$ and satisfies the following estimate for any $t>0$

$$
\|u(t)\|_{L^{2}(\Omega)}^{2} \leq \Lambda e^{-B t}+c \int_{\frac{t}{2}}^{t}\|f(s)\|_{L^{q^{\prime}(\Omega)}}^{q^{\prime}} d s
$$

where $\Lambda=\left\|u_{0}\right\|_{H}^{2}+c\|f\|_{L^{q^{\prime}}\left(\Omega_{\infty}\right)}, c=c\left(q, \nu_{0}, \gamma_{0}\right)$ and $B=B\left(\Omega, N, \nu_{0}\right)$.

Proof of Proposition 3.1. Let $\mathrm{u}$ be a weak energy solution $\mathrm{u}$ of (1.1)-(1.4). Proceeding exactly as in the proof of Theorem 1.1 it follows that $\mathrm{u}$ belongs to $C_{w}([0, T] ; H)$ for any $T>0$.

To prove estimate (3.18), we remark that since $u$ is a global weak energy solution, for every $T>0$ arbitrarily fixed there exists $u_{\varepsilon}$ satisfying Definition 1.2. In particular, thanks to (1.7) the energy equality (1.15) becomes

$$
\begin{aligned}
& \frac{1}{2}\left\|u_{\varepsilon}(t)\right\|_{H}^{2}+\nu_{0} \int_{0}^{t}\left(\left\|D\left(u_{\varepsilon}\right)\right\|_{L^{2}(\Omega)}^{2}+\left\|D\left(u_{\varepsilon}\right)\right\|_{L^{q}(\Omega)}^{q}\right) d s \\
& \quad=\frac{1}{2}\left\|u_{0}\right\|_{H}^{2}+\int_{0}^{t} \int_{\Omega} f: \nabla u_{\varepsilon} d x d s \quad \text { for every } 0 \leq t \leq T .
\end{aligned}
$$


Choosing $t=t_{2}$ in (3.19) and then choosing $t=t_{1}$ again in (3.19) and subtracting the equations obtained in this way we deduce for every $0 \leq t_{1}<t_{2} \leq T$

$$
\begin{aligned}
& \frac{1}{2}\left\|u_{\varepsilon}\left(t_{2}\right)\right\|_{H}^{2}-\frac{1}{2}\left\|u_{\varepsilon}\left(t_{1}\right)\right\|_{H}^{2}+\nu_{0} \int_{t_{1}}^{t_{2}}\left(\left\|D\left(u_{\varepsilon}\right)\right\|_{L^{2}(\Omega)}^{2}+\left\|D\left(u_{\varepsilon}\right)\right\|_{L^{q}(\Omega)}^{q}\right) d s \\
& \quad=\int_{t_{1}}^{t_{2}} \int_{\Omega} f: \nabla u_{\varepsilon} d x d s .
\end{aligned}
$$

By (1.10) and Young inequality, from (3.20) we get

$$
\begin{aligned}
& \frac{1}{2}\left\|u_{\varepsilon}\left(t_{2}\right)\right\|_{H}^{2}-\frac{1}{2}\left\|u_{\varepsilon}\left(t_{1}\right)\right\|_{H}^{2} \\
& \quad+c_{1} \int_{t_{1}}^{t_{2}}\left\|u_{\varepsilon}\right\|_{W^{1,2}(\Omega)}^{2} d s+c_{2} \int_{t_{1}}^{t_{2}}\left\|u_{\varepsilon}\right\|_{W^{1, q}(\Omega)}^{q} d s \\
& \quad \leq c_{3} \int_{t_{1}}^{t_{2}}\|f(s)\|_{L^{q^{\prime}}(\Omega)}^{q^{\prime}} d s .
\end{aligned}
$$

where $c_{1}=c_{1}\left(\nu_{0}\right), c_{2}=c_{2}\left(\nu_{0}, \gamma_{0}\right)$ and $c_{3}=c_{3}\left(q, \nu_{0}, \gamma_{0}\right)$. From the previous inequality we deduce

$$
\begin{aligned}
& \frac{1}{2}\left\|u_{\varepsilon}\left(t_{2}\right)\right\|_{H}^{2}-\frac{1}{2}\left\|u_{\varepsilon}\left(t_{1}\right)\right\|_{H}^{2} \\
& \quad+c_{1} \int_{t_{1}}^{t_{2}}\left\|u_{\varepsilon}\right\|_{W^{1,2}(\Omega)}^{2} d s \leq c_{3} \int_{t_{1}}^{t_{2}}\|f(s)\|_{L^{q^{\prime}}(\Omega)}^{q^{\prime}} d s .
\end{aligned}
$$

The result follows by applying Poincaré inequality and Lemma 2.2.

Remark 3.3. We point out that when $q>2$ estimate (3.18) shows a faster decay (with respect to (1.17)) as $t \rightarrow+\infty$, although it depends on the initial datum $u_{0}$.

\section{Decay estimates for weak limit solutions}

In this section we consider the case of not regular initial data $u_{0} \in H_{1}$ and we prove the following existence result.

Theorem 4.1. Assume that the structural assumptions (1.8), (1.9) and (1.13) hold true a.e. in $\Omega_{\infty}$ with $q>2, g_{1} \in L^{q^{\prime}}\left(\Omega_{T}\right)$ for any $T>0$ and $g_{2} \in L^{1}\left(\Omega_{\infty}\right)$. Let $f \in\left(L^{q^{\prime}}\left(\Omega_{\infty}\right)\right)^{N^{2}}$ and $u_{0} \in H_{1}$. Then there exists a weak limit global solution (wls) $u$ of (1.1)-(1.4). Moreover, for every $\eta>0, u \in L^{\infty}((\eta,+\infty)$; $\left.\left(L^{2}(\Omega)\right)^{N}\right) \cap C_{w}([\eta, \infty) ; H), D u \in L_{l o c}^{q}\left((0,+\infty) ;\left(L^{q}(\Omega)\right)^{N^{2}}\right)$ and for every $0<$ $t_{1}<t_{2}<+\infty$ the following bound holds

$$
\int_{t_{1}}^{t_{2}}\|D u(t)\|_{L^{q}(\Omega)}^{q} \leq \nu_{0}^{-1}\left[\int_{\frac{t_{1}}{2}}^{t_{2}} g(s) d s+\frac{\Lambda_{1}}{t_{1}^{\frac{q}{2}-1}}\right] .
$$

Finally, estimate (1.17) is true for any $t>0$. 
Here, by a weak limit global solution (wls) u of (1.1)-(1.4) we mean a vector function $u$ that for every $T>0$ is a weak limit solution of (1.1)-(1.4), where a weak limit solution $\mathrm{u}$ of (1.1)-(1.4) is a vector-valued function $\mathrm{u}$ in $L_{l o c}^{q}\left((0, T) ; V_{q}\right) \cap L_{l o c}^{\infty}((0, T) ; H)$ satisfying

$$
\begin{aligned}
& -\int_{\Omega_{T}} u \cdot \partial_{t} \varphi d x d t+\int_{\Omega_{T}}(\mathbf{S}(x, t, D(u))-u \otimes u): D(\varphi) d x d t \\
& =\int_{\Omega_{T}} f: \nabla \varphi d x d t
\end{aligned}
$$

for every $\varphi \in C^{\infty}\left(\Omega_{T}\right)^{N}$ with $\nabla \cdot \varphi=0$ and $\operatorname{supp}(\varphi) \subset \subset \Omega \times(0, T)$ and such that $\mathrm{u}$ is the a.e. limit in $\Omega \times(0, T)$ (indeed further convergences hold) of vector functions $u^{\varepsilon} \in C([0, T] ; H)$ satisfying

$$
\lim _{\varepsilon \rightarrow 0}\left\|u^{\varepsilon}(0)-u_{0}\right\|_{L^{1}(\Omega)}=0 .
$$

In other words, $\mathrm{u}$ is a weak limit solution if it is a distributional solution which satisfies the initial condition " $u(0)=u_{0}$ " in the very weak sense stated in (4.3). We point out that in the regular case $u_{0} \in H$, the weak solution constructed in Proposition 2.1 is also a weak limit solution and hence the notion of weak limit solution seems to be a natural extension of the previous notion of weak solution to the more general case of irregular data.

Proof of Theorem 4.1. Let $T>0$ arbitrarily fixed. To prove the existence of a weak limit global solution of $(1.1)-(1.4)$ we approximate the initial datum $u_{0}$. In detail, let $u_{0, \varepsilon} \in H, \varepsilon>0$, such that

$$
u_{0, \varepsilon} \rightarrow u_{0} \quad \text { in }\left(L^{1}(\Omega)\right)^{N} \quad \text { as } \varepsilon \rightarrow 0
$$

Let us consider the sequence $u_{\varepsilon}$, where for every $\varepsilon>0$ the function $u_{\varepsilon}$ is the unique solution belonging to $C([0, T] ; H) \cap L^{q}\left(0, T ; V_{q}\right)$ of the following approximating system

$$
\left\{\begin{array}{lc}
\nabla \cdot u_{\varepsilon}=0 & \text { in } \Omega_{T}, \\
\partial_{t} u_{\varepsilon}+\nabla \cdot\left(u_{\varepsilon} \otimes u_{\varepsilon} \phi_{\varepsilon}\left(\left|u_{\varepsilon}\right|\right)-\mathbf{S}\left(x, t, D\left(u_{\varepsilon}\right)\right)+f\right)=-\nabla p_{\varepsilon} & \text { in } \Omega_{T}, \\
u_{\varepsilon}=0 & \text { on } \partial \Omega \times(0, T), \\
u_{\varepsilon}(0)=u_{0, \varepsilon} & \text { in } \Omega,
\end{array}\right.
$$

where $\phi_{\varepsilon}$ and $p_{\varepsilon}$ are the same functions defined in the proof of Proposition 2.1 .

We observe that, since $u_{\varepsilon}$ is the unique solution of (4.5) which belongs to $C([0, T] ; H) \cap L^{q}\left(0, T ; V_{q}\right)$, it is possible to extend it (in a unique way) to a global solution of (4.5) belonging to $C_{l o c}([0,+\infty) ; H) \cap L_{l o c}^{q}\left([0,+\infty) ; V_{q}\right)$. We denote such a global solution again $u_{\varepsilon}$.

Proceeding exactly as in Sect. 3 for the proof of Theorem 1.1 (see also Remark 3.2) we obtain the following universal estimates

$$
\left\|u_{\varepsilon}(t)\right\|_{L^{2}(\Omega)}^{2} \leq \frac{\Lambda_{1}}{t^{\frac{1}{2}-1}}+\int_{\frac{t}{2}}^{t} g(s) d s \quad \text { for every } t>0
$$




$$
\int_{t_{1}}^{t_{2}}\left\|D u_{\varepsilon}(t)\right\|_{L^{q}(\Omega)}^{q} \leq \nu_{0}^{-1}\left[\int_{\frac{t_{1}}{2}}^{t_{2}} g(s) d s+\frac{\Lambda_{1}}{t_{1}^{\frac{q}{2}-1}}\right] \quad \text { for every } 0<t_{1}<t_{2}<+\infty
$$

where $\Lambda_{1}$ and $g$ are as in the statement of Theorem 1.1. We notice explicitly, that since we are assuming $q>2$ the previous estimates are independent of the initial data $u_{0, \varepsilon}$. This fact allow to proceed exactly as in the proof of Theorem 1.3 in [21] and to conclude that "away from $t=0$ ", i.e. for example for every $0<T_{1}<T$ arbitrarily fixed, there exists a subsequence of $u_{\varepsilon}$ (that we denote $u_{\varepsilon_{1}}$ ) that converges a.e. in $\Omega \times\left(T_{1}, T\right)$ to a function $u \in C_{w}\left(\left[T_{1}, T\right] ; H\right) \cap L^{q}\left(T_{1}, T ; V_{q}\right)$. Moreover, (again by the arguments in the proof of Theorem 1.3 in [21]) stronger convergences hold true which allow to pass to the limit in the approximating problem and to conclude that (4.2) holds true for every test function $\varphi \in C^{\infty}\left(\Omega_{T}\right)^{N}$ with $\nabla \cdot \varphi=0$ and $\operatorname{supp}(\varphi) \subset \subset \Omega \times\left(T_{1}, T\right)$. Let us fix $T_{1}=\frac{T}{2}$. With the same reasoning done above, we can conclude that there exists a subsequence of $u_{\varepsilon_{1}}$ (that we denote $u_{\varepsilon_{2}}$ ) that converges a.e. in $\Omega \times\left(\frac{T}{4}, 2 T\right)$ to a function $v \in C_{w}\left(\left[\frac{T}{4}, 2 T\right] ; H\right) \cap L^{q}\left(\frac{T}{4}, 2 T ; V_{q}\right)$. Moreover, stronger convergences hold true which allow to pass to the limit in the approximating problem and to conclude that (4.2) holds true (with $\mathrm{u}$ replaced by $\mathrm{v}$ and $T$ replaced by $2 T$ ) for every test function $\varphi \in C^{\infty}\left(\Omega_{2 T}\right)^{N}$ with $\nabla \cdot \varphi=0$ and $\operatorname{supp}(\varphi) \subset \subset \Omega \times\left(\frac{T}{4}, 2 T\right)$. We point out that by construction it results $u=v$ in $\Omega \times\left(\frac{T}{2}, T\right)$ and consequently we can denote $u=v$ in all the set $\Omega \times\left(\frac{T}{4}, 2 T\right)$. Iterating this argument in the sets $\Omega \times\left(\frac{T}{2^{n}}, n T\right)$ we can construct a weak limit global solution $\mathrm{u}$ having the stated regularity and satisfying the universal estimate (1.17) for every $t>0$ and the universal bound (3.17) for every $0<t_{1}<t_{2}<+\infty$.

\section{Acknowledgements}

Open access funding provided by Università degli Studi di Roma La Sapienza within the CRUI-CARE Agreement.

Open Access. This article is licensed under a Creative Commons Attribution 4.0 International License, which permits use, sharing, adaptation, distribution and reproduction in any medium or format, as long as you give appropriate credit to the original author(s) and the source, provide a link to the Creative Commons licence, and indicate if changes were made. The images or other third party material in this article are included in the article's Creative Commons licence, unless indicated otherwise in a credit line to the material. If material is not included in the article's Creative Commons licence and your intended use is not permitted by statutory regulation or exceeds the permitted use, you will need to obtain permission directly from the copyright holder. To view a copy of this licence, visit http://creativecommons. org/licenses/by/4.0/.

Publisher's Note Springer Nature remains neutral with regard to jurisdictional claims in published maps and institutional affiliations. 


\section{References}

[1] Astarita, G., Marrucci, G.: Principles of Non-Newtonian Fluid Mechanics. McGraw-Hill, London (1974)

[2] Batchelor, G.K.: An Introduction to Fluid Mechanics. Cambridge University Press, Cambridge (1967)

[3] Bird, R.B., Armstrong, R.C., Hassager, O.: Dynamics of Polymer Liquids, vol. 1: Fluid Mechanics, 2nd edn. Wiley, New York (1987)

[4] Dong, Bo-Qing, Chen, Zhi-Min: Time decay rates of non-Newtonian flows in $\mathbb{R}_{+}^{n}$. J. Math. Anal. Appl. 324, 820-833 (2006)

[5] Diening, L., Rüŭička, M., Wolf, J.: Existence of weak solutions for unsteady motions of generalized Newtonian fluids. Ann. Scuola Norm. Sup. Pisa Cl. Sci. (5) IX, 1-46 (2010)

[6] Evans, L.C.: Partial Differential Equations, Graduate Studies in Mathematics V. 19. American Mathematical Society, Providence (1997)

[7] Farroni, F., Moscariello, G.: A nonlinear parabolic equation with drift term. Nonlinear Anal. TMA 177, 397-412 (2018)

[8] Ladyženskaja, O.: The Mathematical Theory of Viscous Incompressible Flow. Gordon and Beach, New York (1969)

[9] Lamb, H.: Hydrodynamics, 6th edn. Cambridge University Press, Cambridge (1945)

[10] Lions, J.L.: Quelques méthodes de résolution des Problémes aux Limites Non Linéaires. Dunod, Gauthier-Villars, Paris (1969)

[11] Malek, J., Rajagopal, K.R., Rüžička, M.: Existence and regularity of solutions and stability of the rest state for fluids with shear dependent viscosity. Math. Models Methods Appl. 5, 789-812 (1995)

[12] Malek, J., Nečas, J., Ružiča, M.: On weak solutions to a class of non-Newtonian incompressible fluids in bounded three-dimensional domains: the case $\mathrm{p} \geq 2$. Adv. Diff. Equs. 6(3), 257-302 (2001)

[13] Moscariello, G., Porzio, M.M.: Quantitative asymptotic estimates for evolution problems. Nonlinear Anal. TMA 154, 225-240 (2017)

[14] Nečasova, S., Penel, P.: $L^{2}$ decay for weak solutions to equations of NonNewtonian incompressible fluids in the whole space. Nonlinear Anal. 47, 41814192 (2001)

[15] Porzio, M.M.: On decay estimates. J. Evol. Equ. 9(3), 561-591 (2009)

[16] Porzio, M.M.: Existence, uniqueness and behavior of solutions for a class of nonlinear parabolic problems. Nonlinear Anal. TMA 74, 5359-5382 (2011) 
[17] Porzio, M.M.: On uniform and decay estimates for unbounded solutions of partial differential equations. J. Differ. Equ. 259, 6960-7011 (2015)

[18] Porzio, M.M.: Regularity and time behavior of the solutions of linear and quasilinear parabolic equations. Adv. Differ. Equ. 23(5-6), 329-372 (2018)

[19] Schonbek, M.E.: $L^{2}$ decay for weak solutions of the Navier-Stokes equations. Arch. Ration. Mech. Anal. 88, 209-222 (1985)

[20] Wilkinson, W.L.: Non-Newtonian Fluids, Fluid Mechanics, Mixing and Heat Transfer. Pergamon Press, London (1960)

[21] Wolf, J.: Existence of weak solutions to the equations of non-stationary motion of non-Newtonian fluids with shear rate dependent viscosity. J. Math. Fluid Mech. 9, 104-138 (2007)

Gioconda Moscariello

Dipartimento di Matematica e Applicazioni "R. Caccioppoli"

Università degli Studi di Napoli "Federico II"

Via Cintia

80126 Naples

Italy

e-mail: gmoscari@unina.it

Maria Michaela Porzio

Dipartimento di Matematica "G. Castelnuovo"

Sapienza Università di Roma

P.Le A. Moro 2

00185 Rome

Italy

e-mail: mariamichaela.porzio@uniroma1.it

Received: 21 January 2020.

Accepted: 3 July 2020. 